\begin{tabular}{|c|c|c|}
\hline \multicolumn{3}{|c|}{ DJS Vol. 38 (2017) 74- 80} \\
\hline (e) & \multirow{4}{*}{$\begin{array}{c}\text { Delta Journal of Science } \\
\text { Available online at } \\
\text { https://djs.journals.ekb.eg/ }\end{array}$} & Detta Journal of Scierese \\
\hline & & $x_{4} 2$ 父公 \\
\hline 霄 & & $\frac{1}{x-1}$ \\
\hline 1969 & & Sin: \\
\hline \multicolumn{2}{|l|}{ Research Article } & HEMATICS \\
\hline
\end{tabular}

\title{
A Fuzzy Approach for Solving Fractional Programming Problems E.A. Youness ${ }^{1}$, E. F. Ibrahim²,
}

${ }^{I}$ Department of Mathematics Faculty of Science, Tanta University, Tanta, Egypt.

\begin{abstract}
In this paper we describe a fuzzy approach for solving nonlinear fractional programming problem with linear constraints (NLFPP). In the proposed approach, the objective function is transformed into linear function by using Taylor's theorem and the considered NLFPP is changed into equivalent linear programming problem (LPP) which it can be solved as a linear programming problem. The proposed approach is based on choosing three initial points inside the feasible region which enable us to generate a new point at which the value of objective function is better than the previous value, and so on to reach the best approximation of optimal solution.
\end{abstract}

\section{1-Introduction :}

Fuzzy programming is a kind of optimization problems under uncertainty. On the other hand, fuzzy programming considers parameters as fuzzy numbers and constraints are treated as fuzzy sets. Some constraint violation is allowed and the degree of satisfaction of a constraint is defined as the membership function of this constraint.

Many of the developments in the area of fuzzy mathematical programming are based on Bellman and Zadeh [2]. This field has been recently popularized by the work of Zimmermann [9].

Fractional programming (FP), which has been used as an important planning tool for last four decades , is applied to different disciplines such as engineering ,business, economics, Fractional programming is generally used for modeling real life problems with fractional objective such as profit / cost, inventory/sales, actual, cost / standard cost /and output employee etc. [3,5]

The optimization is the science of determining the best solution to certain mathematically defined problems .An objective function defined by a set of decision variables is used to determine the goodness of a solution . the optimal value of the objective function represents the best solution to the problem . Many practical problems can be modeled by an objective function and hence, optimization problems can occur in many areas of research [6].

There are many techniques available for the solution of a constrained

nonlinear programming problems, These techniques like method of Zoutendijk [10,11] and the method introduced by Youness, E.A.,S.Z.Hassan and Y.A. ElRewaily [7] and others, for more details see[1].

\section{2-Problem Formulation and the Solution}

\section{Concept :}

Let us consider the following fractional programming problem can be formulated as:

$\mathrm{E}_{0}$ :

$$
\max F(x)=f(x)+\frac{c^{T} x+\alpha}{d^{T} x+\beta}
$$

Where $d^{T} x+\beta>0$

Subject to:

$$
x \in M
$$

function and

Where $F(x)$ is a real-valued convex objective

$c^{T}, d^{T} \in \mathcal{R}^{n}, \alpha, \beta \in \mathcal{R}$. Furthermore, $\mathrm{M}$ is the feasible region and might be, in the form:

$M=\left\{x \in R^{n} g_{r}(x) \leq 0, x \geq 0,\right\}$,

Where $f(x)$ is a convex function and $g_{r}(x) \leq 0, r=$ $1,2, \ldots, m$ are linear functions.

Let us define a membership function $\mu: M \rightarrow[0,1]$ for the objective function $F(x)$ of (NLFPP) with an aspiration level $\ell$ as follows [4] :

If $F(x) \lesssim \ell$ then

$$
\begin{aligned}
& \mu(x) \\
& =\left\{\begin{array}{cccc}
1 & , & \text { if } & F(x) \leq \ell \\
\bar{t}-F(x) & , & & \\
\bar{t}-\ell & , & \text { if } & \ell \leq F(x) \leq \bar{t} \\
0 & , & \text { if } & F(x) \leq \bar{t}
\end{array}\right.
\end{aligned}
$$

Or if $F(x) \gtrsim \ell$ then

$$
\mu(x)=\left\{\begin{array}{ccc}
1, & \text { if } & F(x) \geq \ell \\
\frac{F(x)-\underline{t}}{\ell-\underline{t}}, & \text { if } & \underline{t} \leq F(x) \leq \ell \\
0, & \text { if } & F(x) \leq \underline{t}
\end{array}\right.
$$


Where $\bar{t}$ and $\underline{t}$ are the upper tolerance and lower tolerance limit for the fuzzy goal for the objective function $F(x)$. By using Zimmermann Max-Min approach [8], the problem $E_{0}$ is transformed into LPP as given below:

$\mathrm{E}_{1}\left\{\begin{array}{c}\operatorname{Max} p_{1}(x)=\tilde{\mu}(x) \cong \mu\left(x^{*}\right)+\sum_{i=1}^{n}\left(x_{i}-x^{*}\right) \frac{\partial \mu\left(x^{*}\right)}{\partial x_{i}} \\ \text { s.t } \\ x \in X=\left\{g_{r}(x) \leq 0, x \geq 0, x^{*} \in \mathcal{R}^{n}\right\}\end{array}\right.$ And

$$
\mu(x)=\left\{\begin{array}{ccc}
1 & \text { if } & F(x) \geq \ell \\
\frac{F(x)-\underline{t}}{\ell-\underline{t}}, & \text { if } & \underline{t} \leq F(x) \leq \ell \\
0, & \text { if } & F(x) \leq \underline{t}
\end{array}\right.
$$

To find the solution of problem $E_{1}$, we formulate a sequence of sub problems $\mathrm{E}_{k}$ in $\alpha \in \mathcal{R}$ as follows.

Choose $x_{k}^{1}, x_{k}^{2}, x_{k}^{3} \in \mathrm{M}$, and calculate the corresponding values of the objective function at these points, $p_{1}\left(x_{k}^{1}\right), p_{1}\left(x_{k}^{2}\right), p_{1}\left(x_{k}^{3}\right)$ and form the function:

$$
\Phi(\alpha)=p_{1}\left(x_{k}^{2}+\alpha\left(x_{k}^{1}-x_{k}^{3}\right)\right)
$$

Consider the order:

$$
p_{1}\left(x_{k}^{1}\right) \leq p_{1}\left(x_{k}^{3}\right) \leq p_{1}\left(x_{k}^{2}\right)
$$

The set of constraints for sub problem $k$ becomes

$$
\begin{gathered}
N=\left\{\alpha \in \mathcal{R}, \Psi_{r}(\alpha)=g_{r}\left(x_{k}^{2}+\alpha\left(x_{k}^{1}-x_{k}^{3}\right)\right) \leq 0,\right. \\
r=1,2, \ldots, m\}
\end{gathered}
$$

For value $\bar{\alpha} \in \mathcal{R}$ which is a solution to the sub problems $\mathrm{E}_{k}$,

$$
\mathrm{E}_{k}\left\{\begin{array}{c}
\operatorname{Max} \Phi(\alpha) \\
\text { s.t } \\
N=\left\{\alpha \in \mathcal{R}, \Psi_{r}(\alpha)=g_{r}\left(x_{k}^{2}+\alpha\left(x_{k}^{1}-x_{k}^{3}\right)\right)\right. \\
\leq 0, r=1,2, \ldots, m\}
\end{array}\right.
$$

We obtain anew point $\overline{x_{k+1}}=x_{k}^{2}+\bar{\alpha}\left(x_{k}^{1}-x_{k}^{3}\right)$, we can exclude one point, of the three points chosen before, that corresponding the minimum value of $E_{k}$

\section{3-Main results}

Lemma1 : if $M \subset R^{n}$ is a convex set then for any $\overline{x_{k}^{1}, x_{k}^{2}, x_{k}^{3}} \in M$,

$N=\left\{\alpha \in R: \Psi_{r}(\alpha)=g_{r}\left(x_{k}^{2}+\alpha\left(x_{k}^{1}-x_{k}^{3}\right)\right) \leq\right.$ $0, r=1,2, \ldots, m\}$ is a convex

\section{Proof:}

Let $\bar{\alpha}, \widehat{\alpha} \in \mathcal{R}, 0 \leq \lambda \leq 1$

$$
\text { Then } \begin{aligned}
& \Psi_{r}(\lambda \bar{\alpha}+(1-\lambda) \hat{\alpha}) \\
= & g_{r}\left(x_{k}^{2}+(\lambda \bar{\alpha}+(1-\lambda) \hat{\alpha})\left(x_{k}^{1}-x_{k}^{3}\right)\right)
\end{aligned}
$$

$$
\begin{aligned}
& =g_{r}\left(\begin{array}{c}
x_{k}^{2}+\lambda \bar{\alpha}\left(x_{k}^{1}-x_{k}^{3}\right)+ \\
(1-\lambda) \hat{\alpha}\left(x_{k}^{1}-x_{k}^{3}\right)
\end{array}\right) \\
& =g_{r}\left(\begin{array}{c}
\lambda\left(x_{k}^{2}+\bar{\alpha}\left(x_{k}^{1}-x_{k}^{3}\right)\right)+ \\
(1-\lambda) \hat{\alpha}\left(x_{k}^{2}+\left(x_{k}^{1}-x_{k}^{3}\right)\right.
\end{array}\right)
\end{aligned}
$$

Since $x_{k}^{2}+\bar{\alpha}\left(x_{k}^{1}-x_{k}^{3}\right) \in M$, then from linearity of $g_{r}$ we have

$$
\Psi_{r}(\lambda \bar{\alpha}+(1-\lambda) \hat{\alpha})=\lambda g_{r}\left(\left(x_{k}^{2}+\right.\right.
$$

$\left.\bar{\alpha}\left(x_{k}^{1}-x_{k}^{3}\right)\right)+(1-\lambda) g_{r}\left(x_{k}^{2}+\left(x_{k}^{1}-x_{k}^{3}\right)\right)=$ $\lambda \Psi_{r}(\bar{\alpha})+(1-\lambda) \Psi_{r}(\hat{\alpha}) \leq 0$

So $\mathrm{N}$ is a convex

Lemma 2: If $\bar{\alpha} \in N$ is the solution for the problem $\mathrm{E}_{1}$ then :

$\phi(\bar{\alpha}) \geq \mathrm{p}\left(\mathrm{x}_{\mathrm{i}}\right), \mathrm{i}=1,2,3$.

\section{Proof :}

Since $\bar{\alpha} \in \mathcal{R}$ is the solution (maximum) for the problem $\mathrm{E}_{1}$

$$
\phi(\bar{\alpha}) \geq \mathrm{p}(\alpha), \forall \alpha \in \mathrm{N}
$$

i.e.

$$
p\left(x_{k}^{2}+\bar{\alpha}\left(x_{k}^{1}-x_{k}^{3}\right)\right) \geq p\left(x_{k}^{2}+\alpha\left(x_{k}^{1}-x_{k}^{3}\right), \forall \alpha\right.
$$

Where $0 \in N$ then

$$
p\left(x_{k}^{2}+\bar{\alpha}\left(x_{k}^{1}-x_{k}^{3}\right)\right) \geq p\left(x_{k}^{2}\right) \geq p\left(x_{k}^{1}\right) \geq p\left(x_{k}^{3}\right)
$$

Lemma 3 : If $\bar{\alpha}=0 \in N$ is the solution (maximal point) for the problem $\mathrm{E}_{1}$ and $\hat{a} \in N$ is the minimal point for the problem $\mathrm{E}_{1}$ then : $\quad \phi(\widehat{\alpha}) \geq$ $\min _{i} p\left(x_{i}\right), i=1,2,3$.

\section{Proof:}

Assume $x_{t} \in\left\{x_{1}, x_{2}, x_{3}\right\}$ such that

$$
p\left(x_{t}\right)=\min _{i} p\left(x_{i}\right), i=1,2,3
$$

We consider two the following problems:

$$
\begin{gathered}
E_{1} \quad \begin{array}{c}
\operatorname{Min} \Phi(\alpha) \\
\alpha \in N
\end{array} \\
E_{2} \quad\left\{\begin{array}{c}
\operatorname{Min} \Phi(\alpha) \\
\alpha \in N \\
\Phi(\alpha) \geq p\left(x_{t}\right)
\end{array}\right.
\end{gathered}
$$

Let $\hat{a}$ be the solution of $E_{2}$ then we will prove that $\hat{a}$ is a solution of $E_{1}$

Suppose that $\hat{\alpha}$ isn't a solution of the problem $E_{1}^{\prime}$ then there exists $\alpha^{*} \in N$ Such that $\Phi\left(\alpha^{*}\right)<\Phi(\hat{\alpha})$ but 
$\Phi\left(\alpha^{*}\right) \geq p\left(x_{t}\right)$, which contradicts that $\hat{\alpha}$ is the solution of $E_{1}$. So $\Phi(\hat{\alpha})$ is satisfied that

$$
\phi(\hat{a}) \geq \min _{i} f\left(x_{i}\right), i=1,2,3
$$

\section{Lemma 4:}

If $\mu\left(\overline{x_{k}}\right)=1$ or, $\mu\left(\overline{x_{k+1}}\right)<\mu\left(\overline{x_{k}}\right), F\left(\overline{x_{k+1}}\right)<$ $F\left(\overline{x_{k}}\right)$, then then there is no a solution of $\mathrm{E}_{0}$ different from $\overline{x_{k}}$

\section{Proof :}

Suppose that $\hat{x} \in N$, is a solution of $\mathrm{E}_{0}$ then

$$
F(\hat{x})>F\left(\overline{x_{l}}\right), \mathrm{i}=1,2,3 \ldots \mathrm{k}
$$

And from the definition of the membership function , we get

$$
\mu(\hat{x})>\mu\left(\bar{x}_{l}\right), \mathrm{i}=1,2,3 \ldots, k
$$

So $\hat{x}$ represent $\overline{x_{k+1}}$ hence $\mu(\hat{x})=\mu\left(\overline{x_{k+1}}\right)>$ $\mu\left(\overline{x_{l}}\right), \mathrm{i}=1,2,3 \ldots, k$

Which is a contradiction .Hence, there is no a solution of $\mathrm{E}_{0}$ different from $\overline{x_{k}}$

\section{4-The Algorithm}

Can be explained in these steps :

Step 1: Determine the vertices of the feasible region, $x^{*}=\left\{x_{1}^{*}, x_{2}^{*}, \ldots, x_{n}^{*}\right\}$ for the objective function $F(x)$ where $n$ is the number of variable

Step 2: Calculate the upper tolerance and lower tolerance limit $\bar{t}$ and $\underline{t}$ for the fuzzy goal for the objective function $F(x)$

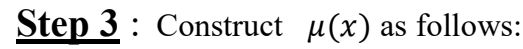

if $F(x) \lesssim \ell$ then

$$
\begin{aligned}
& \mu(x) \\
& =\left\{\begin{array}{lllc}
1 & , & \text { if } & F(x) \leq \ell \\
\hline \bar{t}-F(x) & & & \\
\bar{t}-\ell & , & \text { if } & \ell \leq F(x) \leq \bar{t} \\
0 & , & \text { if } & F(x) \leq \bar{t}
\end{array}\right.
\end{aligned}
$$

Or if $F(x) \gtrsim \ell$ then

$$
\begin{aligned}
& \mu(x) \\
& =\left\{\begin{array}{ccc}
1 & \text { if } & F(x) \geq \ell \\
\frac{F(x)-\underline{t}}{\ell-\underline{t},} & \text { if } & \underline{t} \leq F(x) \leq \ell \\
0, & \text { if } & F(x) \leq \underline{t}
\end{array}\right.
\end{aligned}
$$

Step 4 : Transform The problem into the form

$$
\mathrm{E}_{1}\left\{\begin{array}{l}
p_{1}(x) \cong \mu\left(x^{*}\right)+\sum_{i=1}^{n}\left(x_{i}-x^{*}\right) \frac{\partial \mu\left(x^{*}\right)}{\partial x_{i}} \\
\quad s . t \\
x \in X=\left\{g_{r}(x) \leq 0, x \geq 0, x^{*} \in \mathcal{R}^{m}\right.
\end{array}\right.
$$

\section{Step 5:}

Let $I_{1}=\left\{x_{1}, x_{2}, x_{3}\right\} \subset M$ then calculate $p_{1}\left(x_{i}\right), i=1,2,3$ where $p_{1}\left(x_{1}\right)<p_{1}\left(x_{3}\right)<p_{1}\left(x_{2}\right)$

\section{$\underline{\text { Step 6: }}$}

Let $\bar{x}=x_{2}+\alpha\left(x_{1}-x_{3}\right)$ then form

$\Phi(\alpha)=p_{1}\left[x_{2}+\alpha\left(x_{1}-x_{3}\right)\right]$ and function

$\Psi_{r}(\alpha)=g_{r}\left[x_{2}+\alpha\left(x_{1}-x_{3}\right)\right], r=1,2,3, \ldots, m$.

\section{Step 7:}

Solve

$\mathrm{E}_{k}\left\{\begin{array}{c}\operatorname{Max} \Phi(\alpha) \\ \text { s.t } \\ N=\left\{\alpha \in \mathcal{R}, \Psi_{r}(\alpha)=g_{r}\left(x_{k}^{2}+\alpha\left(x_{k}^{1}-x_{k}^{3}\right)\right) \leq 0\right. \\ , r=1,2, \ldots, m\}\end{array}\right.$

Let $\bar{\alpha}$ is the solution

\section{Step 8:}

Change $x_{2} \in I_{1}$ where $p_{1}\left(x_{2}\right)>p_{1}\left(x_{i}\right)$ with

$\bar{x}=x_{2}+\alpha\left(x_{1}-x_{3}\right)$ and $f(\bar{x})>f\left(x_{2}\right)$,

$\mu(\bar{x})>\mu\left(x_{2}\right)$ then we obtained $I_{2}$ and go to step 4

If $\alpha=0$ then go to step 9

\section{Step 9:}

Determine $\operatorname{Min} \Phi(\alpha)$ on $N$, let $\alpha=\hat{\alpha}$ then change $x_{2} \in I_{1}$ where $p_{1}\left(x_{2}\right)>p_{1}\left(x_{i}\right)$ with $\hat{x}=x_{2}+$ $\hat{\alpha}\left(x_{1}-x_{3}\right)$ then we obtained $I_{2}$ and go to step 6

Step 10: (stopping point)

$x_{2}^{k} \in I_{k}$ where $p_{1}\left(x_{2}^{k}\right)>p_{1}\left(x_{i}^{k}\right)$ with $\overline{x_{k+1}}=x_{2}^{k}+$ $\alpha\left(x_{1}^{k}-x_{3}^{k}\right)$ and $\mu\left(\overline{x_{k+1}}\right)<\mu\left(\overline{x_{k}}\right), f\left(\overline{x_{k+1}}\right)<$ $f\left(\overline{x_{k}}\right)$ then stop

Or if $\mu\left(\overline{x_{k+1}}\right)=1$ then stop

\section{5-An Illustrative Example :}

In this section, illustrative examples are given to clarify the proposed solution algorithm

The problems to be solved here are the following Nonlinear programming problems by using a fuzzy approach: [1] 
$\operatorname{Max} f(x)=x_{1}^{2}+x_{2}^{2}+\frac{2 x_{1}-3 x_{2}-5}{x_{1}+1}$

s.t

$$
\begin{gathered}
x_{1} \leq 6 \\
x_{2} \leq 6 \\
2 x_{1}+x_{2} \leq 9, \\
-2 x_{1}+x_{2} \leq 5, \\
x_{1}-x_{2} \leq 5, \\
x_{1}, x_{2} \geq 0,
\end{gathered}
$$

The solution steps of this problem due to the algorithm indicated as follows:

If the fuzzy aspiration level of the objective function is -5 then find $x$ to satisfy the following fuzzy goal,

$$
f(x) \gtrsim-5
$$

The tolerance limit for the fuzzy goal is 30.25 respectively, the membership function for the fuzzy goal is :

$$
\begin{aligned}
& \mu(x) \\
& =\left\{\begin{array}{ccc}
1 & F(x)-\underline{t} \\
\ell-\underline{t}, & \text { if } & F(x) \geq \ell \\
0, & \text { if } & \underline{t} \leq F(x) \leq \ell
\end{array}\right.
\end{aligned}
$$$$
\text { i.e }
$$

\begin{tabular}{|c|c|c|c|c|c|}
\hline Iteration & Points & $\begin{aligned} & \boldsymbol{p}(\boldsymbol{x}) \\
& \boldsymbol{p}\left(x_{1}\right) \\
&<p\left(x_{3}\right) \\
&<p\left(x_{2}\right)\end{aligned}$ & $\begin{array}{c}\bar{x}= \\
x_{2}+ \\
\alpha\left(x_{3}-\right. \\
\left.x_{1}\right)\end{array}$ & $F(\bar{x})$ & $\boldsymbol{\mu}(\overline{\boldsymbol{x}})$ \\
\hline$I_{1}$ & $\begin{array}{l}x_{1} \\
=(1,5) \\
x_{2} \\
=(0.5,6) \\
x_{3} \\
=(2,5)\end{array}$ & $\begin{array}{l}\boldsymbol{p}\left(x_{1}\right) \\
=0.5943 \\
\boldsymbol{p}\left(x_{2}\right) \\
=0.8014 \\
\boldsymbol{p}\left(x_{3}\right) \\
=\mathbf{0 . 7 9 2 9}\end{array}$ & $(1.5,6)$ & 30.25 & 1 \\
\hline
\end{tabular}

$$
\begin{gathered}
\mu(x) \\
=\left\{\begin{array}{lcc}
1, & \text { if } \quad F(x) \geq 30.25 \\
\frac{x_{1}^{2}+x_{2}^{2}+\frac{2 x_{1}-3 x_{2}-5}{x_{1}+1}+5}{35.25}, & \text { if }-5 \leq F(x) \leq 30.25 \\
0, & \text { if } & F(x) \leq-5
\end{array}\right. \\
\mu(x) \cong \mu(1.5,6)+\left(x_{1}-1.5\right) \frac{\partial \mu(1.5,6)}{\partial x_{1}}+\left(x_{2}\right. \\
-6) \frac{\partial \mu(1.5,6)}{\partial x_{2}} \\
\mu(x) \cong 0.1986 x_{1}+0.3064 x_{2}-1.1363
\end{gathered}
$$

\begin{tabular}{|c|c|c|c|c|c|}
\hline Iteration & Points & $\begin{array}{l}\boldsymbol{p}\left(x_{1}\right) \\
<p\left(x_{3}\right) \\
<p\left(x_{2}\right)\end{array}$ & $\begin{array}{c}\bar{x}= \\
x_{2}+ \\
\alpha\left(x_{1}-x_{3}\right) \\
\end{array}$ & $F(\bar{x})$ & $\mu(\bar{x})$ \\
\hline$I_{1}$ & $\begin{array}{c}x_{1}=(4.5,1) \\
x_{2}=(2.8,3.4) \\
x_{3}=(0,5)\end{array}$ & $\begin{array}{l}\boldsymbol{p}\left(x_{1}\right) \\
=\mathbf{0 . 0 6 3 8} \\
\boldsymbol{p}\left(x_{2}\right) \\
=\mathbf{0 . 4 6 1 5} \\
\boldsymbol{p}\left(x_{3}\right) \\
=\mathbf{0 . 3 9 5 7} \\
\end{array}$ & $(0.307,5.616)$ & 15.387 & 0.58 \\
\hline$I_{2}$ & $\begin{array}{c}x_{1}=(0,5) \\
x_{2} \\
=(0.307,5.616) \\
x_{3}=(2.8,3.4)\end{array}$ & $\begin{array}{l}\boldsymbol{p}\left(x_{1}\right) \\
=\mathbf{0 . 3 9 5 7} \\
\boldsymbol{p}\left(x_{2}\right) \\
=0.645 \\
\boldsymbol{p}\left(x_{3}\right) \\
=\mathbf{0 . 4 6 1 5}\end{array}$ & $(2.246,4.508)$ & 21.044 & 0.74 \\
\hline$I_{3}$ & $\begin{array}{l}x_{1}=(2.8,3.4) \\
x_{2} \\
=(2.246,4.508) \\
x_{3} \\
=(0.307,5.616)\end{array}$ & $\begin{array}{l}\boldsymbol{p}\left(x_{1}\right) \\
=\mathbf{0 . 4 6 1 5} \\
\boldsymbol{p}\left(x_{2}\right) \\
=0.691 \\
\boldsymbol{p}\left(x_{3}\right) \\
=\mathbf{0 . 6 4 5}\end{array}$ & $(0.568,5.999)$ & 22.369 & 0.78 \\
\hline$I_{4}$ & $\begin{array}{l}x_{1} \\
=(0.307,5.616) \\
x_{2} \\
=(0.568,5.999) \\
x_{3} \\
=(2.246,4.508)\end{array}$ & $\begin{array}{l}p\left(x_{1}\right) \\
=0.645 \\
\boldsymbol{p}\left(x_{2}\right) \\
=0.8146 \\
\boldsymbol{p}\left(x_{3}\right) \\
=\mathbf{0 . 6 9 1}\end{array}$ & $(1.873,5.25)$ & 25.152 & 0.86 \\
\hline$I_{5}$ & $\begin{array}{l}x_{1} \\
=(2.246,4.508) \\
x_{2} \\
=(1.873,5.25) \\
x_{3} \\
=(0.568,5.999)\end{array}$ & $\begin{array}{l}\boldsymbol{p}\left(x_{1}\right) \\
=\mathbf{0 . 6 9 1} \\
\boldsymbol{p}\left(x_{2}\right) \\
=0.844 \\
\boldsymbol{p}\left(x_{3}\right) \\
=\mathbf{0 . 8 1 4 6}\end{array}$ & $(1.03,5.999)$ & 27.09 & 0.9 \\
\hline$I_{6}$ & $\begin{array}{l}x_{1} \\
=(0.568,5.999) \\
x_{2} \\
=(1.03,5.999) \\
x_{3} \\
=(1.873,5.25)\end{array}$ & $\begin{array}{l}\boldsymbol{p}\left(x_{1}\right) \\
=\mathbf{0 . 8 1 4 6} \\
\boldsymbol{p}\left(x_{2}\right) \\
=\mathbf{0} .906 \\
\boldsymbol{p}\left(x_{3}\right) \\
=0.844\end{array}$ & $(1.714,5.615)$ & 27.65 & 0.926 \\
\hline
\end{tabular}

We get LPP:

$$
\operatorname{Max} p(x)=0.1986 x_{1}+0.3064 x_{2}-1.1363
$$

s.t

$$
\begin{gathered}
x_{1} \leq 6, \\
x_{2} \leq 6, \\
2 x_{1}+x_{2} \leq 9, \\
-x_{1}+x_{2} \leq 5,
\end{gathered}
$$

$$
x_{1}-x_{2} \leq 5
$$

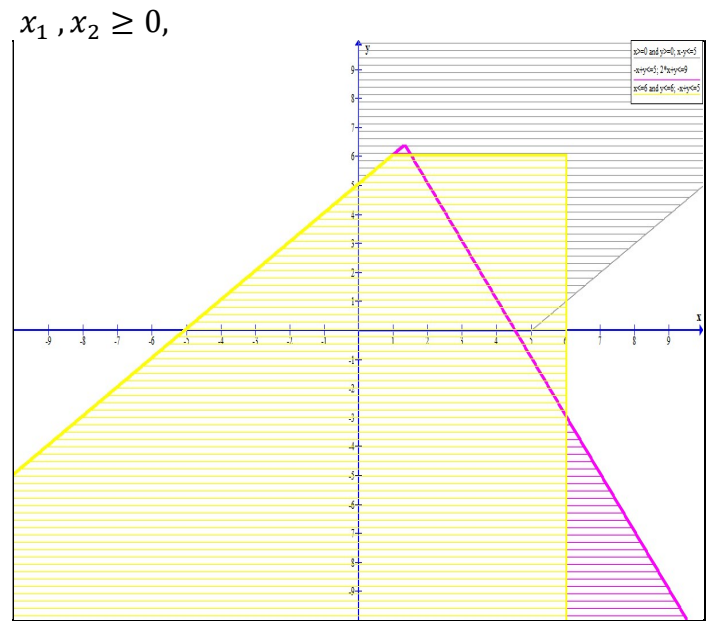

Table 1 the result of illustrative example

In table1we observed $\mu(\bar{x})=1$ so the optimum maximum value is 30.25 at $\left(x_{1} x_{2}\right)=(1.5,6)$.

Table 2 the result of illustrative example 


\begin{tabular}{|c|c|c|c|c|c|}
\hline$I_{7}$ & $\begin{array}{l}x_{1} \\
=(1.873,5.25) \\
x_{2} \\
=(1.714,5.615) \\
x_{3} \\
=(1.03,5.999)\end{array}$ & $\begin{array}{l}\boldsymbol{p}\left(x_{1}\right) \\
=0.844 \\
p\left(x_{2}\right) \\
=0.924 \\
\boldsymbol{p}\left(x_{3}\right) \\
=\mathbf{0} .906 \\
\end{array}$ & $(1.28,5.99)$ & 28.57 & 0.952 \\
\hline$I_{8}$ & $\begin{array}{l}x_{1} \\
=(1.03,5.999) \\
x_{2}=(1.28,5.99) \\
x_{3} \\
=(1.714,5.615)\end{array}$ & $\begin{array}{l}\boldsymbol{p}\left(x_{1}\right) \\
=\mathbf{0} .906 \\
p\left(x_{2}\right) \\
=0.952 \\
\boldsymbol{p}\left(x_{3}\right) \\
=0.924\end{array}$ & $(1.585,5.829)$ & 29.017 & 0.96 \\
\hline$I_{19}$ & $\begin{array}{l}x_{1} \\
=(1.5126,5.975) \\
x_{2} \\
=(1.5098,5.98) \\
x_{3} \\
=(1.714,5.615)\end{array}$ & $\begin{array}{l}\boldsymbol{p}\left(x_{1}\right) \\
=\mathbf{0} \\
p\left(x_{2}\right) \\
=0.9958 \\
\boldsymbol{p}\left(x_{3}\right) \\
=0.9954\end{array}$ & $(1.4815,5.999)$ & 30.1116 & 0.996 \\
\hline$I_{20}$ & $\begin{array}{l}x_{1} \\
=(1.714,5.615) \\
x_{2} \\
=(1.4815,5.999) \\
x_{3} \\
=(1.5098,5.98)\end{array}$ & $\begin{array}{l}\boldsymbol{p}\left(x_{1}\right) \\
=0.9954 \\
p\left(x_{2}\right) \\
=0.996 \\
\boldsymbol{p}\left(x_{3}\right) \\
=0.9958\end{array}$ & $(1.48,5.9999)$ & 30.1086 & 0.9957 \\
\hline
\end{tabular}

In table 2 we observed $\mu\left(\overline{x_{20}}\right)<\mu\left(\overline{x_{19}}\right), f\left(\overline{x_{20}}\right)<$ $f\left(\overline{x_{19}}\right)$ then stop,

So the optimum maximum value is 30.1116 at $\left(x_{1} x_{2}\right)=(1.4815,5.999)$.

\section{6-Conclusion:}

This paper has discussed a fuzzy approach for solving nonlinear fractional programming problem with linear constraints. By using Taylor's theorem the NLFPP is converted into equivalent linear programming problem (LPP) which it can be solved as a linear programming problem. The presented algorithm has fined the point at which the value of objective function, based on choosing three initial points inside the feasible region which enable us to generate a new point at which the value of objective function is better than the previous value, and so on to reach the best approximation of optimal solution.

\section{References:}

[1] Bazaraa, M. S., Sherali, H. D. (2006) Nonlinear Programming Theory and Algorithms, 3rd Edition .

[2] Bellman, R. \& Zadeh, L.A. (1970). DecisionMaking in a Fuzzy Environment, Management Science , 17: 141-161.

[3] Frenk, J.B.G. and Schaible, S., (2001). Fractional Programming: Introduction and Applications: In: Encyclopedia, Floudas, C. A. and Pardalos, P.M. (Eds.). Kluwer Academic Publishers. Dordrecht. Netherlands, 162-172.

[4] Pitam Singh, Shiv Datt Kumar and R. K. Singh( 2010) . Fuzzy Method for Multi objecive Linear Plus Linear Fractional Programming Problem ,International Mathematical ,60:2971-2983.

[5] Schaible, S. and Shi, J. (2004). Recent Developments in Fractional Programming: Single Ratio and Max-min Case, Working Paper Series, 04-02, A. Gary Anderson Graduate School of Management, University of California, Riverside, CA 92521, USA.

[6] Stephens,c.(1997).Global optimization theory versus practice , $\mathrm{PhD}$ thesis, University of Canterbury, Christchurch, New Zealand

[7] Youness, E.A., Hassan ,S.Z. and El-Rewaily, Y.A. (2008). Quadratic Interpolation Algorithm for Minimizing Tabulated Function, Journal of Mathematics and Statistics4 ,4:217-221.

[8] Zimmermann, H. J. (1978). Fuzzy programming and linear programming with several objective functions, Fuzzy sets and systems, 1: 45-55.

[9] Zimmermann, H. J.(1991). Fuzzy Set Theory and its Application, $2^{\text {nd }}$ Ed., Kluwer Academic Publishers, Boston.

[10]Zoutendijk, G. (1960). Methods of Feasible Directions, Elsevier, Amsterdam, and D. Van Nostrand, Princeton, NJ

[11]Zoutendijk, G. (1976). Mathematical Programming Methods, North-Holland, Amsterdam, The Netherlands. 


\section{ملخص البحث}

هذالبحث يقدم طريقة فازية لحل مشكلة البرمجة الكسرية تحت قيود خطية .هذه الطريقة تقوم على تحويل مشكلة البرمجة الكسرية إلى مشكلة خطية باستخدام نظرية تايلور وحلها عن طريق اختيار ثلاث نقاط داخل منطقة الحلول المتاحة ثم توليد نقطة جديدة تحقق أكبر قيمة ويتجاهل النقطة التي تحقق أقل قيمة وتكرر هذه العملية حتى نصل إلى أفضل الحلول تقريبا أو الحل الأمثل. 